\title{
Line It Up - Inadvertent Placement of Nasogastric Tube in Pleural Space Resulting in Iatrogenic Empyema
}

\author{
Murtaza Hussain ${ }^{1}$, Smit Deliwala $^{1}$, Dominic Awuah ${ }^{1}$, and Aashish Valvani ${ }^{1}$ \\ ${ }^{1}$ Hurley Medical Center
}

July 3, 2021

\begin{abstract}
Dobhoff tubes, used for post-pyloric feedings, have a weighted metal end with a small diameter that enhances their flexibility to traverse the gastrointestinal tract. Unfortunately, the metal stylet can iatrogenically perforate surrounding structures in patients with diminished cough and gag (1); and extreme caution should be considered before its utilization.
\end{abstract}

Line It Up - Inadvertent Placement of Nasogastric Tube in Pleural Space Resulting in Iatrogenic Empyema

Murtaza S. Hussain, $\mathrm{MD}^{1}$, Smit S. Deliwala, $\mathrm{MD}^{1}$, Rohit Gupta, $\mathrm{MD}^{2}$, Aashish Valvani $\mathrm{MD}^{3}$

${ }^{1}$ Department of Internal Medicine, Michigan State University at Hurley Medical Center, Flint, MI

2 Department of Internal Medicine and Pediatrics, Michigan State University at Hurley Medical Center, Flint, MI

3 Division of Pulmonary/Critical Care, Department of Internal Medicine, Michigan State University at Hurley Medical Center, Flint, MI

Correspondence:

Murtaza S. Hussain, MD

Hurley Medical Center

One Hurley Plaza

Flint, MI - 48503

USA

\section{Key Clinical Message/Abstract:}

Dobhoff tubes, used for post-pyloric feedings, have a weighted metal end with a small diameter that enhances their flexibility to traverse the gastrointestinal tract. Unfortunately, the metal stylet can iatrogenically perforate surrounding structures in patients with diminished cough and gag (1); and extreme caution should be considered before its utilization.

Key Words: Nasogastric tube; feeding tube; malposition; perforation

Case History: 
Post-pyloric feedings are an essential strategy in critically ill patients to facilitate tube feed delivery and mitigate aspiration events. As with other feeding modes, Dobhoff tubes also require a clinical and radiologic confirmation for proper placement.

Question: What are the implications of incorrect Dobhoff tube placement?

Answer: Dobhoff tubes can easily perforate vital surrounding structures and placement should be confirmed.

A 66-year-old male with several comorbidities presented with altered mental status and septic shock requiring a prolonged stay in the intensive care unit. A nasogastric tube was placed and confirmed via visual observation on consecutive abdominal radiographs (Figure 1, Figure 2); tube feeding was subsequently initiated. Within a few hours, the patient began experiencing desaturation episodes with an emergent computed tomography (CT) of the chest and abdomen (Figure 3, Figure 4), revealing a new left hydropneumothorax and pleural effusion from iatrogenic puncture of pleura. Tube feedings were stopped and a chest tube was placed immediately with drainage and eventual seal.

\section{Author Contributions:}

Murtaza S. Hussain - Acquisition, draft, and review

Smit S. Deliwala - Conception, draft, and review

Dominic Awuah - Acquisition and review

Rohit Gupta - Acquisition and review

Conflict of Interest : None to declare

Funding : None to declare

\section{References:}

1. Lo JO, Wu V, Reh D, Nadig S, Wax MK. Diagnosis and Management of a Misplaced Nasogastric Tube Into the Pulmonary Pleura. Arch Otolaryngol Head Neck Surg. 2008;134(5):547-550

2. Abidali A, Mangram A, Shirah GR, Wilson W, Abidali A, Moeser P, et al. Bilateral pneumothoraces in a trauma patient after Dobhoff tube insertion. The American journal of case reports. 2018;19:244. 

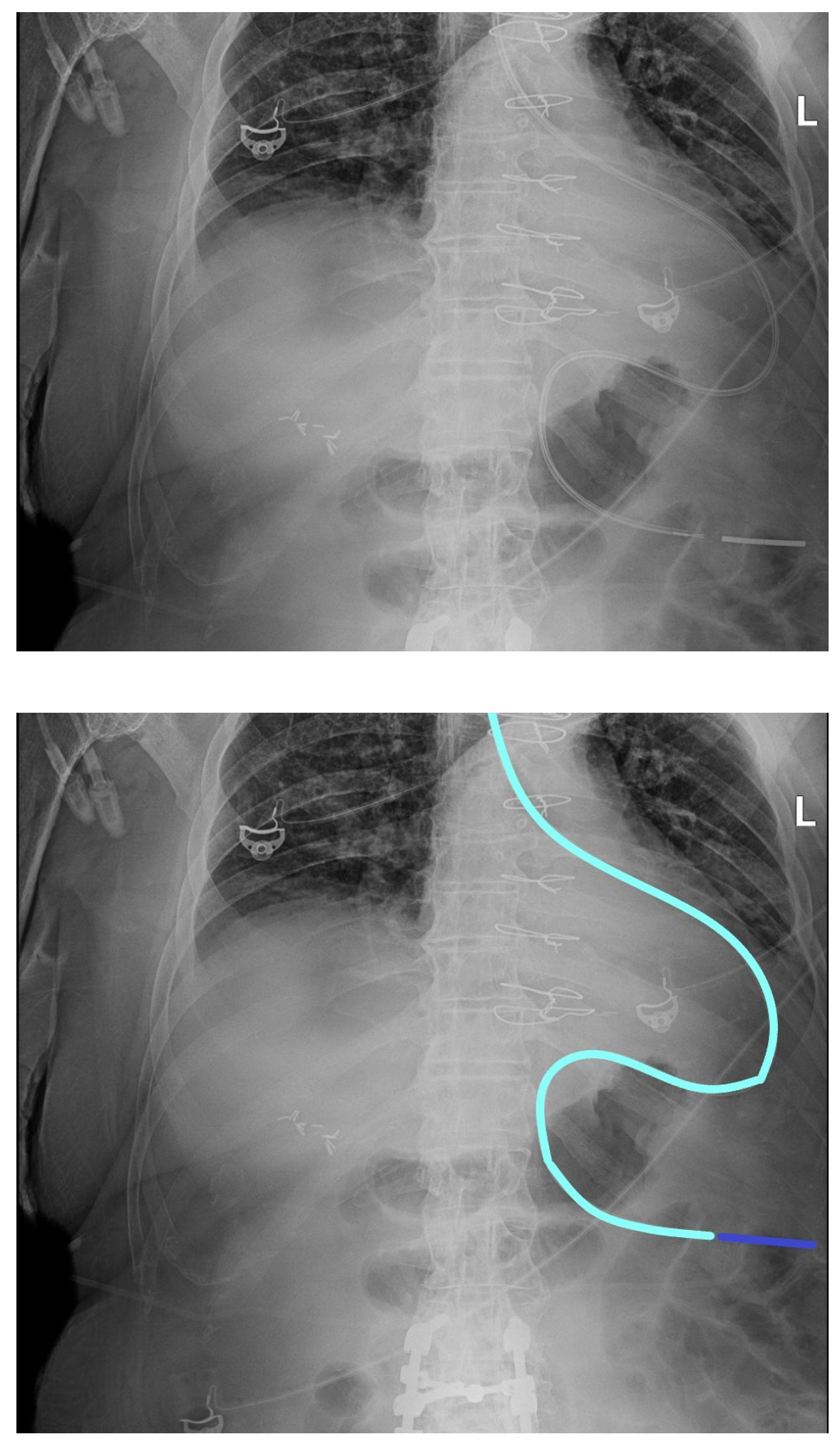

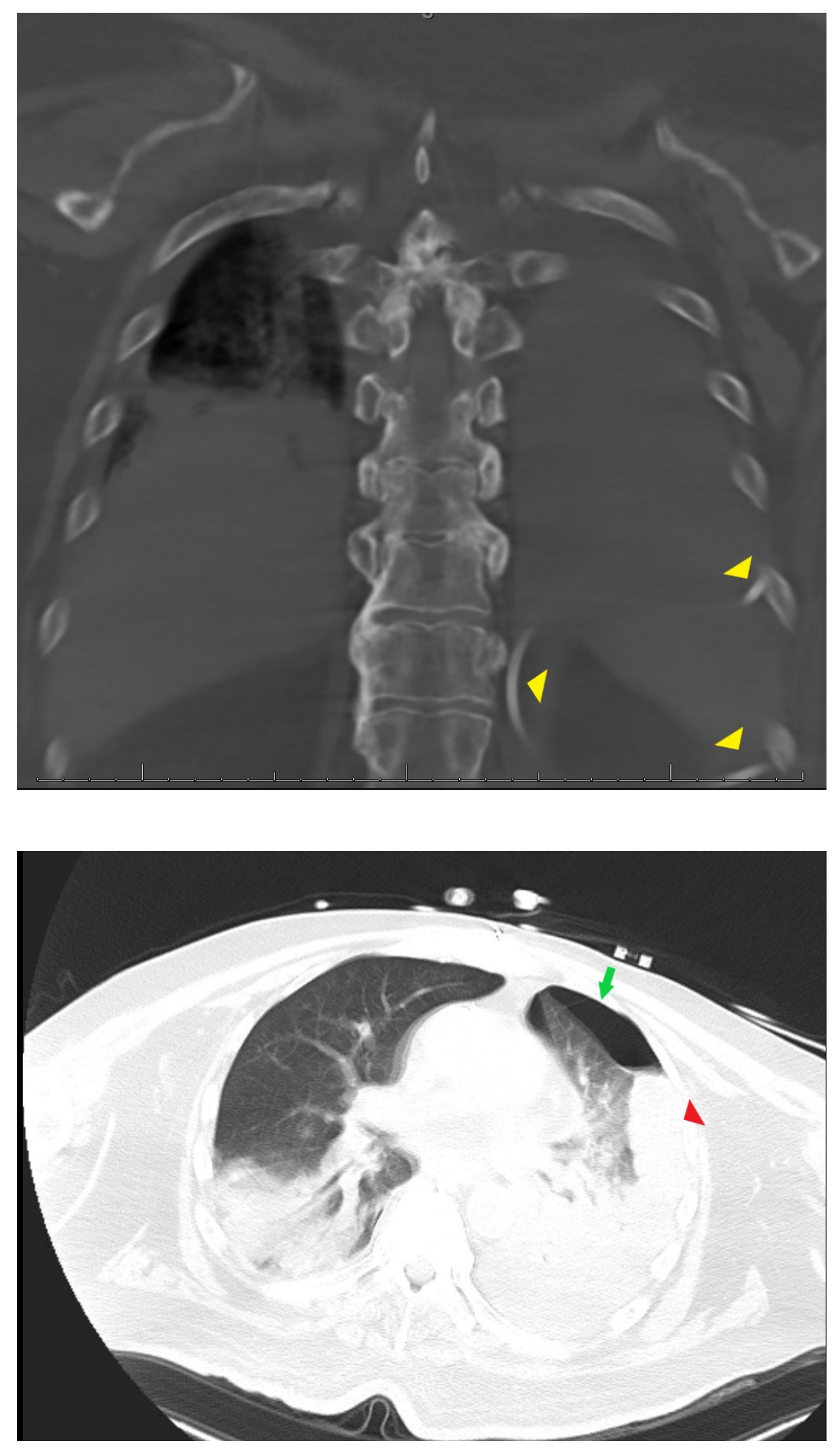